\title{
Cancer in Saudi Arabia (CSA): Web-Based Application to Study Cancer Data Among Saudis Using Waterfall Model
}

\author{
Suliman A Alsagaby (iD) \\ Mafawez T Alharbi (D) ${ }^{2}$ \\ 'Department of Medical Laboratories \\ Sciences, College of Applied Medical \\ Sciences, Majmaah University, Majmaah, \\ I 1952, Saudi Arabia; ${ }^{2}$ Department of \\ Natural and Applied Sciences, \\ Community College, Qassim University, \\ Buraydah, Saudi Arabia
}

Background: Information technology (IT) has emerged as a promising enabler to address the issue of big data in health care. Despite the urgent need for an IT-based tool to tackle this issue, one is not available to specifically study the massive data related to cancer among Saudis.

Objective: To develop a web-based application, which we named "Cancer in Saudi Arabia (CSA)" to provide an interactive, quick, and easy method to reach, extract, compare, and visualize cancer data from Saudi Cancer Incidence Reports (SCIRs).

Methods: We used waterfall model to develop CSA. Next, we used CSA to study the data of non-Hodgkin lymphoma (NHL) in Saudis reported in the SCIRs (1999-2015).

Results: CSA-based analysis showed that NHL incidence rate increased with age and the disease was more common among males compared with females. In addition, NHL was most predominant in the regions of Riyadh and Eastern, while it was the least prevalent in Jazan Region. Interestingly, the largest proportion of NHL patients was diagnosed in the late stage, and malignant lymphoma, large B-cell diffuse, OS (DLBCL) were the most frequent subtypes of NHL.

Conclusion: As a user-friendly application, we believe that CSA will be a useful tool for studying cancer data in Saudis and will make the data published in SCIRs more reachable and usable. Our findings of NHL provided an almost comprehensive view of the epidemiology of the disease in Saudis for 17 years.

Keywords: information technology, cancer in Saudis, epidemiology, big data, waterfall model

\section{Introduction}

Big data in health care refers to patient-related data sets that are very large and complex. ${ }^{1}$ The massive size and complexity of these data present a great challenge to extract useful clues about a disease in a community. ${ }^{2}$ Therefore, this limits the construction of knowledge needed to take an evidence-based action to tackle an illness. Information technology (IT) has appeared as a promising enabler to address the issue of big data in health care. ${ }^{3}$ Consequently, IT applications are very handy to provide a mean by which the complex and big data can be simplified, visualized, and studied in a timeeffective manner. This has caused many observers to believe that IT will improve the ability of health care systems to deliver a better service for public health. ${ }^{4}$

Cancer is one of the most leading causes of death worldwide. Globally, the number of new cases with cancer has been estimated to reach 19.3 million with 10 million
Correspondence: Mafawez T Alharbi; Suliman A Alsagaby Email maft.alharbi@qu.edu.sa; s.alsaqaby@mu.edu.sa 
deaths caused by cancer in the year of $2020 .^{5}$ In the United States, it has been estimated that $1,806,590$ patients will be diagnosed with cancer in 2020 and that 606,520 patients will die of cancer in the same year. ${ }^{6}$ According to the most recent (SCIR) that was published in September 2018, there were 16,210 (50 of 100,000 individuals) newly diagnosed patients with cancer in Saudi Arabia in 2015. ${ }^{7}$ With a life-threatening disease such as cancer, the availability of long-term clinical data is essential to construct accurate knowledge of the illness in order to fight it.

SCIRs are authored and published by the Saudi Cancer Registry (SCR), and downloadable through the SCR web page (https://nhic.gov.sa/en/eServices/Pages/TumorRegistration. aspx). The first SCIR was published on May 1996 and the most recent was published in September 2018. The total number of SCIRs published thus far is 18. Each of the first three SCIRs covered cancer data from multiple years, while the rest contained data from single years. SCIR reports information related to five topics: age-specific incidence rate (AIR) of cancer, regional distribution of age-standardized rate (ASR) of cancer incidents, ASR of cancer incidents among Saudis and other nationalities, stage distribution of cancer, and morphological distribution of cancer. SCIRs have been published in a non-interactive format (portable document format; PDF) averaging approximately 100 pages for each report (1800 pages for all the published SCIRs). The total number of cancer patients whose data have been reported in the 18 SCIRs is 238,634. This brief description demonstrates the complexity of the SCIRs and the huge quantity of data that the reports contain. Therefore, this has restricted taking full advantage of SCIRs, although they contain important knowledge about cancer in Saudi Arabia. Consequently, there has been an urgent need for an interactive, easy, and time-effective means to study SCIRs data.

IT holds a promise as enablers to easily and timeeffectively study and manage big data in health care. ${ }^{3}$ Various web-based applications have been constructed to allow a fast, intuitive, and interactive method for working with cancer data. For example, the cancer statistics data visualizations tool (CSDVT; https://gis.cdc.gov/Cancer/ USCS/DataViz.html) accommodates American-related cancer data resourced from the American Center for Disease Control and Prevention (CDC) and the National Cancer Institute (NCI). ${ }^{19}$ Another example is CancerData (https://www.cancerdata.nhs.uk/) that presents cancer data of patients living in England, which are gathered from the National Cancer Registration and Analysis Service (NCRAS). ${ }^{20}$ Encouraged by these two examples and due to the urgent demand for a simple and quick method to study Saudi cancer data in the SCIRs, the objective of this current work was to employ IT to develop a web-based tool, which we named "Cancer in Saudi Arabia (CSA)" (https://cancerinsaudi.com/). Next, we used the data of non-Hodgkin lymphoma (NHL), which is a group of hematological malignancies, in Saudi patients to explain how CSA works and to provide a nearly complete view of the NHL epidemiology for 17 years (1999-2015).

\section{Methods}

We followed the methodology of software engineering to build CSA website. ${ }^{8}$ The Waterfall Model has been used to build and develop the CSA website. ${ }^{9}$ The waterfall model has been chosen for this research for the following reasons (waterfall advantages): This model is a sequential process arranged from top to bottom in specific stages, as it goes downward through the phases of requirements, the model begins and ends one stage before starting the next one, it is not overlapping stages. Each stage is completed in specified period of time after that it moves to next stage. This approach ensures project deadline control. The model ensures the design flaws of the proposed system before the development of the final product. This is because requirement is clear before development starts. The model supports intensive documentation and planning, where documentations are very useful to use it in the future for code modification and the proposed system is described as high-quality control. It provides an early detection of faults as each stage is clearly defined so, this process ensures early detection of faults.

The proposed system which use this model can be described as: faster, cheaper, better, more flexible, and adaptive. ${ }^{10}$ Our work consisted of five phases: requirements, design, implementation, testing and maintenance.

\section{Phase I: Requirements}

This phase involves the process of discovering the goal of CSA, which is to provide a user-friendly tool to study cancer data in Saudis. Four main tasks were set to be conducted by CSA; reaching, extraction, comparison, and visualization of SCIRs data. This phase addresses the functional requirements of these four tasks. We studied the SCIRs and found that five major topics were reported in each SCIR; therefore, we have selected them:

- Age-Specific Incidence Rate (AIR) of Cancer 
- Regional Distribution of the Age-Standardized Rate (ASR) of Cancer incidents

- AIR of Cancer Incidents among Saudis and Other Nationalities

- Stage Distribution of Cancer

- Morphological Distribution of Cancer

The data related to the above topics were extracted from the SCIRs to construct five tables.

\section{Phase 2: Design}

To design the SCA website, we first created the tables of the system. Each table is a separate entity and the only connection among all the tables is the year. A full description of these tables is shown in Tables 1Tables 2Tables 3Tables 4-5. To describe the SCA website, we used Unified Modeling Language (UML) tool. ${ }^{11}$ UML enabled the visualization of the SCA design. Using UML, we constructed the entity relationship diagram (ERD) and use case of CSA. ERD described the relationships of the tables needed for CSA (Figure 1). These tables had attributes that define their properties.

There are two types of user for CSA, the public and the admin user. The public user can browse the website and view all the pages; reach, extract, compare, and visualize SCIRs

Table I Age-Specific Incidence Rate (AIR)

\begin{tabular}{|l|l|}
\hline Name of the Field & Type of the Inserted Data \\
\hline Disease type & Character \\
\hline Year & Date \\
\hline All Ages Or Total & Number \\
\hline Age Unk & Number \\
\hline Crud Rate & Number \\
\hline ASR World & Number \\
\hline Age Range Values & Number \\
\hline
\end{tabular}

Table 2 Morphological Distribution of Cancer

\begin{tabular}{|l|l|}
\hline Name of the Field & Type of the Inserted Data \\
\hline Disease name & Character \\
\hline Year & Date \\
\hline Histology (Morphology) & Number \\
\hline
\end{tabular}

Table 3 Stage Distribution of Cancer

\begin{tabular}{|l|l|}
\hline Name of the Field & Type of the Inserted Data \\
\hline Disease name & Character \\
\hline Year & Date \\
\hline Stage & Number \\
\hline
\end{tabular}

Table 4 Regional Distribution of Age-Standardized Rate (ASR) of Cancer Incidence in Saudis

\begin{tabular}{|l|l|}
\hline Name of the Field & Type of the Inserted Data \\
\hline Disease name & Character \\
\hline Year & Date \\
\hline Regional Values & Number \\
\hline
\end{tabular}

Table 5 Age-Standardized Rate (ASR) of Cancer Incidence in Saudis and Other Nationalities

\begin{tabular}{|l|l|}
\hline Name of the Field & Type of the Inserted Data \\
\hline Disease name & Character \\
\hline Year & Date \\
\hline Country Values & Number \\
\hline
\end{tabular}

data related to one or more types of cancer for single or multiple years. The second use case is for the admin, who manages and controls the website. The admin can add new cancer data and receive feedback from public users. The admin needs user name and password to access the control panel.

\section{Phase 3: Implementation}

Various tools, which were divided into back end and front end, were used to execute the SCA. The back end tools used Hypertext Preprocessor (PHP), which is designed for web development. ${ }^{12}$ In addition, Laravel was used as the framework for the web development application. ${ }^{13}$ For the front end tools, Hyper Text Markup Language (HTML) was used to create pages and make them functional; Cascading Style Sheet (CSS) was employed to describe how HTML elements can be presented on screen; Java script was utilized to allow access to interactive web pages; and Vue.js was used as a JavaScript framework for setting up and designing user interfaces and single page applications. ${ }^{14-16}$ As a result of the implementation, the SCA website was built. There are two types of 


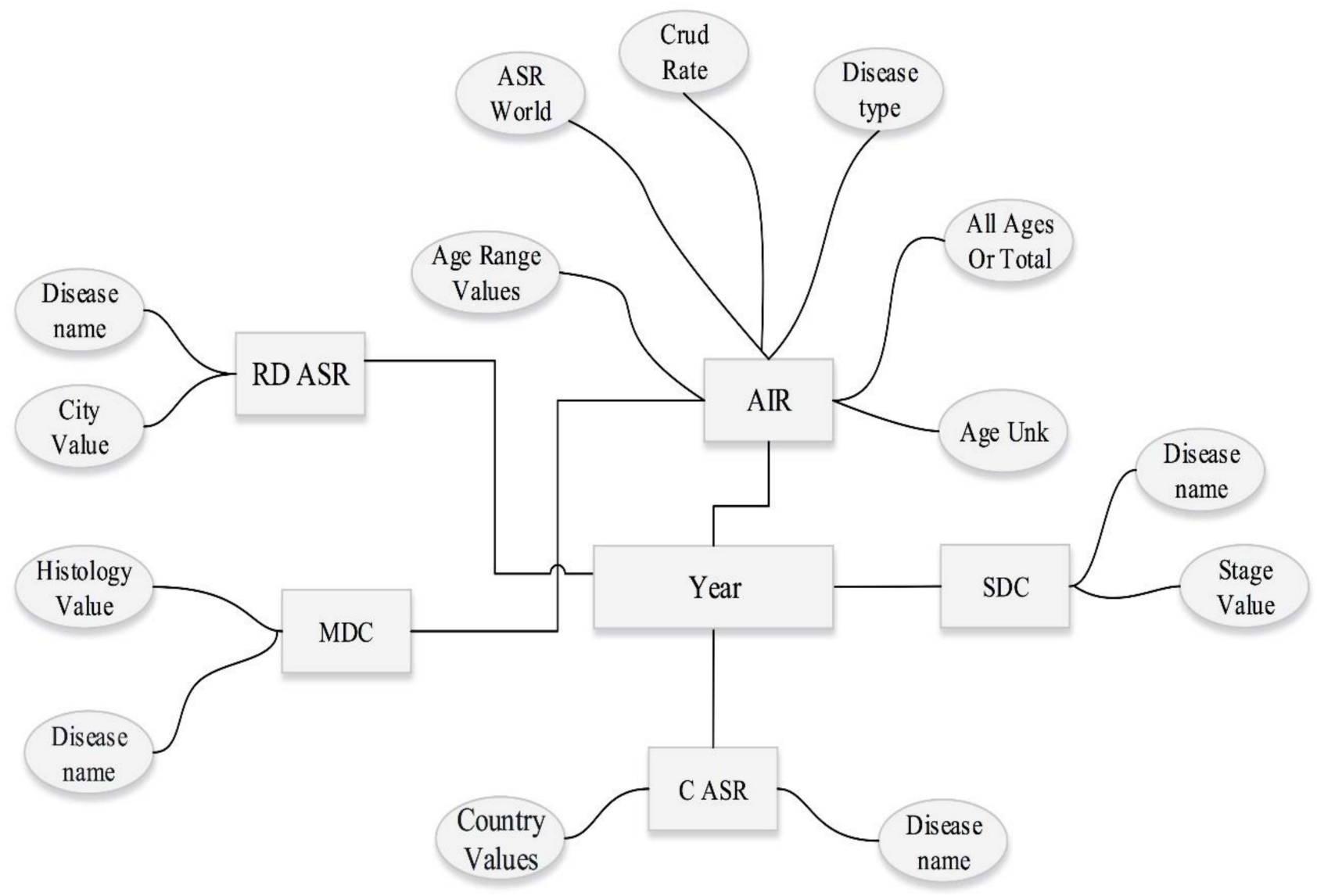

Figure I Entity relationship diagram (ERD) of CSA. ERD shows the relationship between the tables.

Abbreviations: RD ASR, regional distribution of age-standardized rate of cancer incidence; MDC, morphology distribution of cancer; C ASR, country ASR of cancer incidence; SDC, stage distribution of cancer; AIR, age-specific incidence rate.

interfaces in CSA: one for the admin who has control over the system, and the other for the public who wants to use the system. The main admin page is shown in Supplementary Figure 1, through which the admin can $\log$ in to the control panel. There are many tasks that can be performed via this page. The admin page contains subpages, such as those shown in Supplementary Figures 2-6 which the admin uses in order to update/insert related data.

\section{Phase 4: Testing}

The main purpose for this phase is to make sure that the final CSA website, meets its goal. This was be done through examining:

- The ability of the final version of the system to perform the tasks that have been set in Phase 1 .

- The correctness of the inserted data. A double check has been conducted by comparing the original data with the data inserted in the SCA (Supplementary Figure 7).

- The nonfunctional requirements of our system, such as:

1. Security: The CSA website is secure as it follows the standards for writing code which will prevent any threat by hackers. Supplementary Figure 8 shows that the URL starts with https.

2. Usability: We have built CSA to be intuitive and user-friendly. We also made CSA available in English and Arabic languages.

3. Availability: CSA is available because the host server is powerful.

\section{Phase 5: Maintenance}

This phase involves any change or improvements that can be applied to the CSA website after being delivered to public users. Some changes might occur in CSA when effective software systems require updates and amendments over time. The authors will utilize public users' 
feedback that can be received through the "contact us icon" (Supplementary Figure 9).

\section{Results}

\section{Home-Page}

CSA (https://cancerinsaudi.com/) is an interactive, userfriendly and time-effective web-based tool that allows users to reach, extract, compare, and visualize Saudi cancer data in
SCIRs. The home page of CSA is shown in Figure 2. CSA allows the analyses to be conducted according to user preferences with respect to age range, sex, year, region, country, cancer stage, and cancer morphology. CSA also enables users to export the analysis output either in a PDF or TIFF formatted file. Furthermore, the original data underpinning an analysis can be exported to an Excel file. We have built CSA to support both English and Arabic languages to make it more usable.
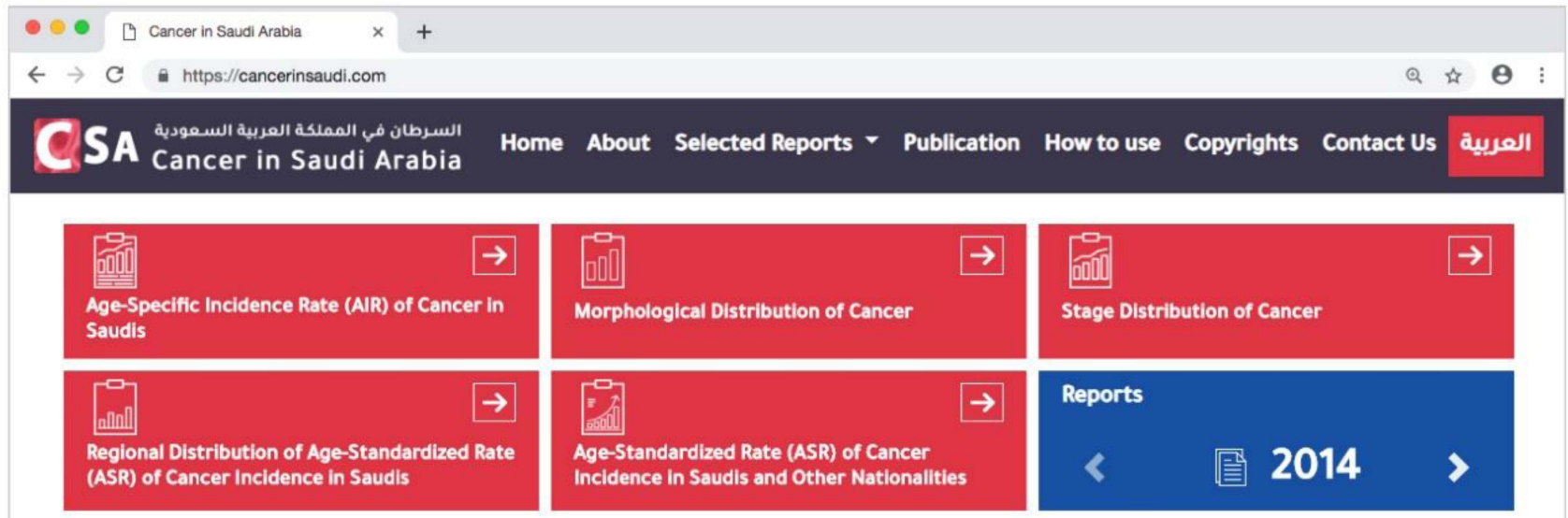

Figure 2 Homepage of the Cancer in Saudi Arabia (CSA).
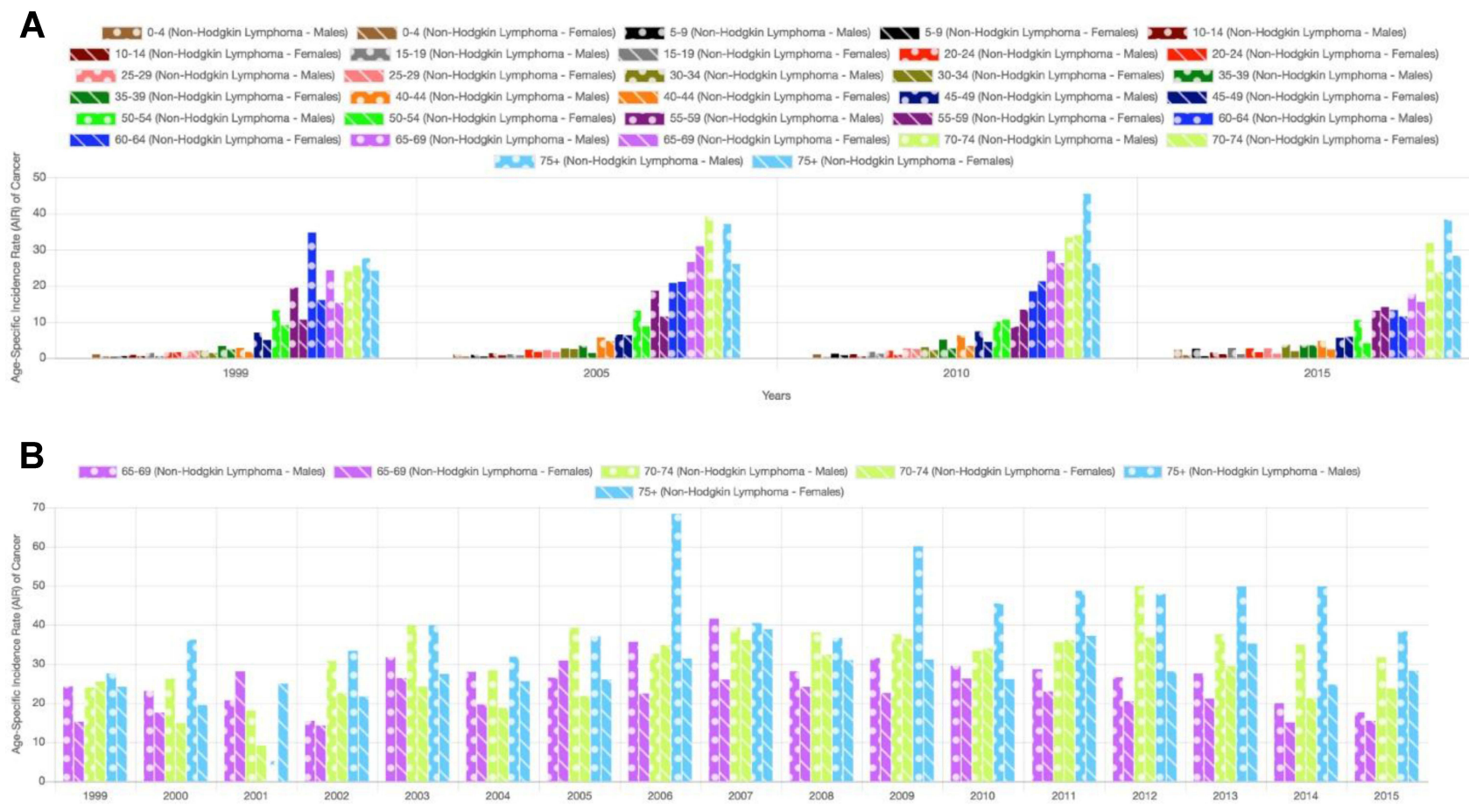

Figure 3 Age specific incidence rate (AIR) of cancer in Saudis per 100,000 population. (A) Comparison of AIR data of all age groups data that were reported in four selected years (only for years were used for simplicity). (B) The AIR data of the top three old age groups from all the 17 years were compared. Asterisk symbol was used to indicate non-available data from SCIRs. 


\section{AIR of Cancer in Saudis}

The age specific incidence rate (AIR) is the number of cancer cases of a specific age and sex at a particular time divided by the total number of population with the same age and sex at midyear. AIR is reported per 100,000 population in the SCIRs. Therefore, AIR is widely used measure to indicate the prevalence of cancer in a specific gender and age group. The comparison of AIR data from 16 age groups in four selected years showed the risk of developing NHL increases with age (Figure 3A). With few exceptions, NHL cases occurred more among male patients compared with female patients. Next, we compared the AIR data of the oldest three age groups from 1999 to 2015 (Figure 3B). Overall, the disease appeared to be predominant among patients at age $\geq 75$; the highest AIR data were recorded at the age group $\geq 75$ for 10 years in men and for 8 years in women. Furthermore, the male predisposition to NHL was more evident in the age group $\geq 75$. The data suggested a trend of decrease of NHL cases in patients at the age group 65-69 starting from 2007 to 2015 and in the age group of 70-74 from 2012 to 2015 .

\section{Regional Distribution of ASR of Cancer Incidence in Saudis}

SCIRs provided data about the cancer incidents among Saudis from the different regions of Saudi Arabia. In this comparison, ASR was used rather than the AIR. ASR is a means of measurement used to compare different populations with variable age structure. ASR employs a standardized age structure known as the "world standard population" and the ASR values are expressed per 100,000 population. The total number of Saudi regions of which ASR data of cancer incidents were reported in SCIRs was 13 . Figure 4A shows a CSA-based comparison of NHL ASR data of Saudis in 13 Saudi regions over seven selected years. Four regions, Riyadh, Najran, Jouf and Eastern, showed high ASR value in both sexes (ASR $\geq 8$ ) in one or more years. In contrast, only the regions of Jazan and Baha recorded low ASR numbers (ASR $<3$ ) in one or both sexes for all 7 years. Next, the ASR data of these six regions from 1999 to 2015 were studied (Figure 4B). Riyadh region reported the largest ASR of NHL in men for seven years. The Eastern region was second to Riyadh, where ASR values of NHL incidents in males were the largest in four years. To the contrary, the
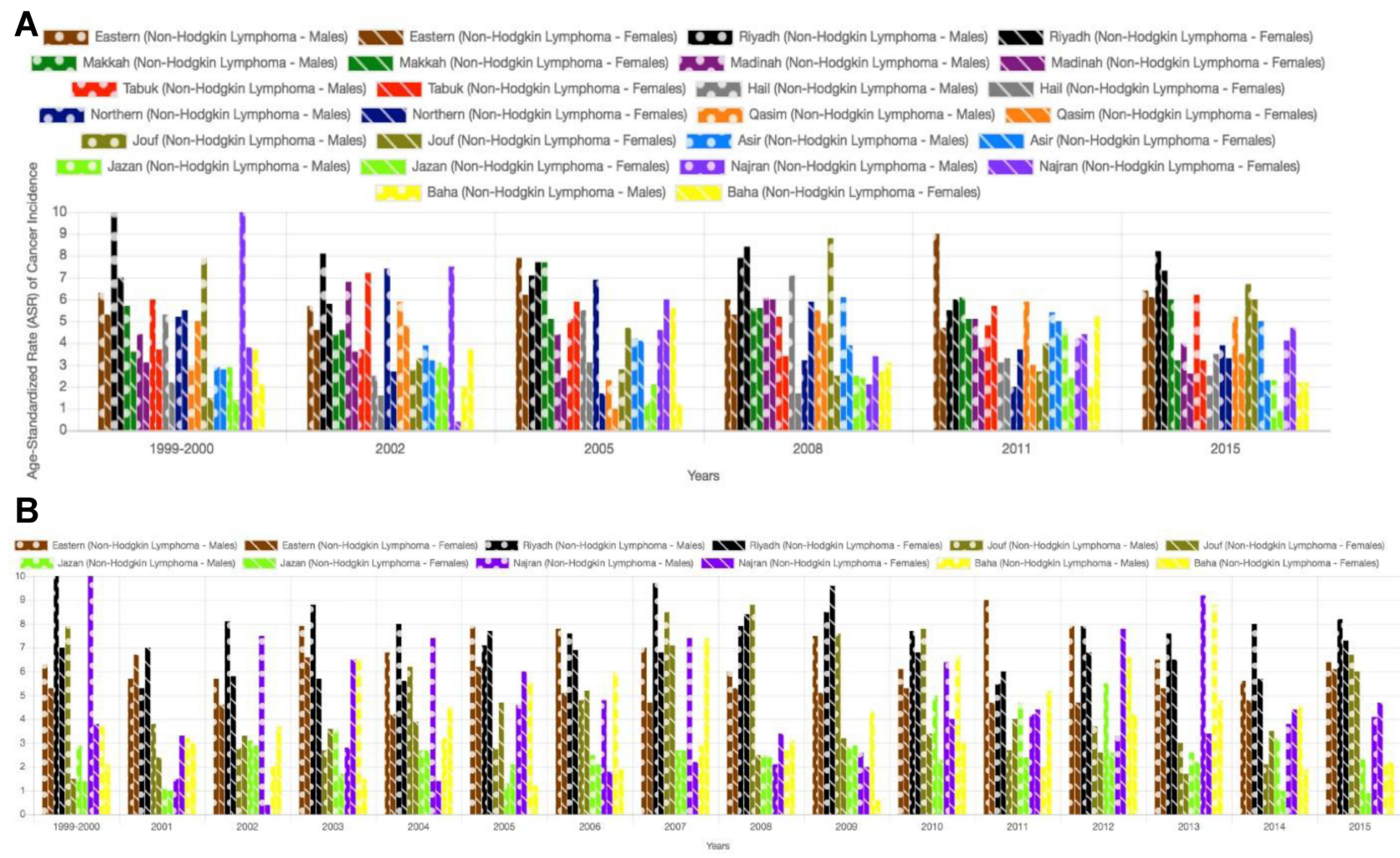

Figure 4 Age standardized rate (ASR) of NHL in Saudis per 100,000 population. (A) The ASR data of all the 13 Saudi regions from seven selected years (only seven years were used for simplicity) were compared using CSA tool. (B) A comparison of the ASR data of six selected regions of Saudi Arabia that were reported in the I7 years. 
lowest ASR data of male NHL was recorded in the region of Jazan for seven years. With the exception of the years 2003, 2007 and 2012, the Riyadh region reported the highest ASR of NHL among females (Figure 4B). In contrast, the lowest ASR of female NHL was recorded in the Jazan region for eight years.

\section{ASR of Cancer Incidents Among Saudis and Other Nationalities}

To gain an insight into the cancer incidents in Saudi Arabia and other countries, SCIRs provide data of the ASR of cancer cases in Saudi Arabia as well as other countries. The total number of the countries for which ASR was reported in the SCIRs (1999-2015) was 56. Nevertheless, not all of these counties appeared in every SCIR. The US, Oman, Bahrain, Kuwait and United Arab Emirates were reported in the majority of the SCIRs. We compared the ASR data in these countries with that of Saudi Arabia (Figure 5). The US claimed the highest ASR of NHL. The highest ASR of male NHL among the Arabic countries was from Kuwait for eight years. In contrast, Saudi Arabia and the United Arab Emirates (UAE) reported the lowest ASR of male NHL for five and eight years, respectively. For females with NHL, Kuwait recorded the highest ASR for 12 years compared with the other Arabic countries. Saudi Arabia and the UAE had the lowest ASR of female NHL for five and four years, respectively. The male to female propensity for NHL in the majority of the years was more evident in the US and Kuwait.

\section{Stage Distribution of Cancer}

In stage distribution of cancer, SCIRs grouped patients on the basis of disease extent into five categories: distant, localized, regional, in situ, and unknown, as defined by SEER. ${ }^{17}$ Stage distribution of cancer is given in percentage rather than number of patients in SCIRs. The category "in situ" is not applicable to NHL; therefore, it was omitted from the analysis. The stage distribution of male and female NHL is very similar, so only NHL data from men are studied here for simplicity. Evidently, the dominant stage of NHL among Saudi patients is the distance (Figure 6). There was a trend of decrease in the patients diagnosed with the unknown stage after 2005. Nevertheless, the proportion of localized stage showed a trend of increase in the last five years (2011-2015). Unlike other stages, the regional stage did not exceed $21 \%$ throughout the 17 years (1999-2015).

\section{Morphological Distribution of Cancer}

In the SCIRs, the morphology (histology or subtype) of cancer cases were identified and reported in accordance with the international classification of diseases for oncology (ICD-O-2) and (ICD-O-3). ${ }^{18}$ We compared 13 morphologies of NHL that were reported in the majority of the SCIRs (1999-2015; Figure 7). Interestingly, malignant lymphoma, diffuse large B-cell, NOS (DLBCL) by far dominated all the other morphologies. Noticeably, the number of cases denoted the morphology "others" raised between 2011 and 2015 after a sudden drop after 2001. The other morphologies were much less frequent in NHL patients ( $\leq 41$ cases) in almost all years (2001-2015). Among the less common morphologies, Burkitt's Lymphoma (BL) was highly reported in males (Figure 8A), whereas malignant lymphoma, non-Hodgkin, NOS was the most common among females (Figure 8B).

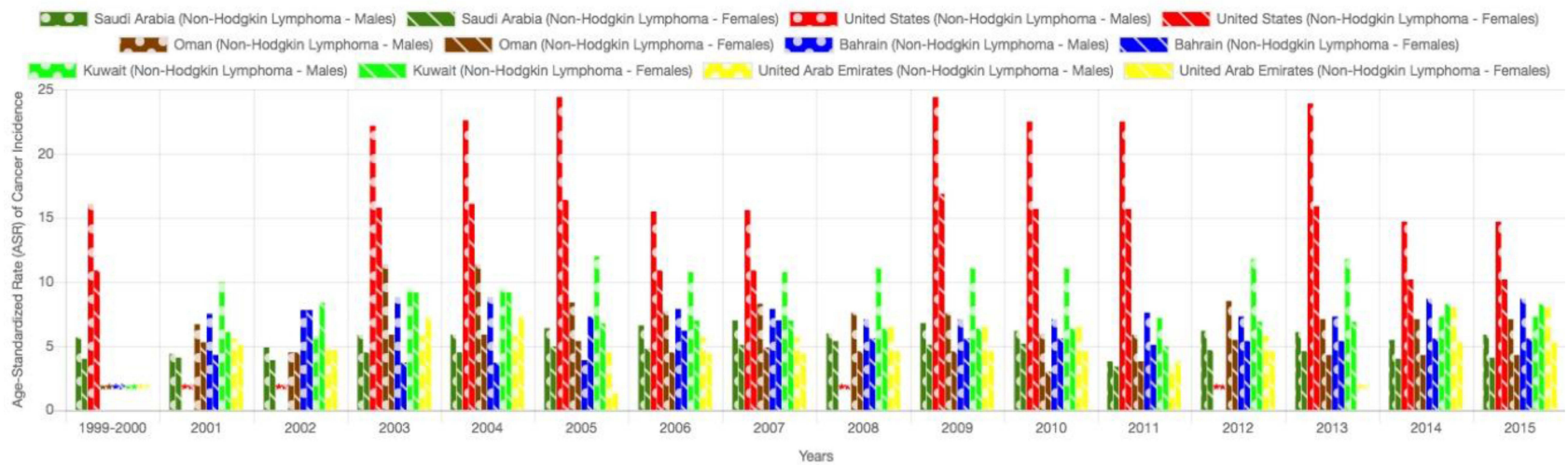

Figure 5 Age standardized rate (ASR) of NHL in Saudis and other nationalities. The ASR of NHL from Saudi Arabia and other Arabic countries along with the US were compared. Asterisk symbol was used to indicate non-available data from SCIRs. Asterisk symbol was used to indicate non-available data from SCIRs. 


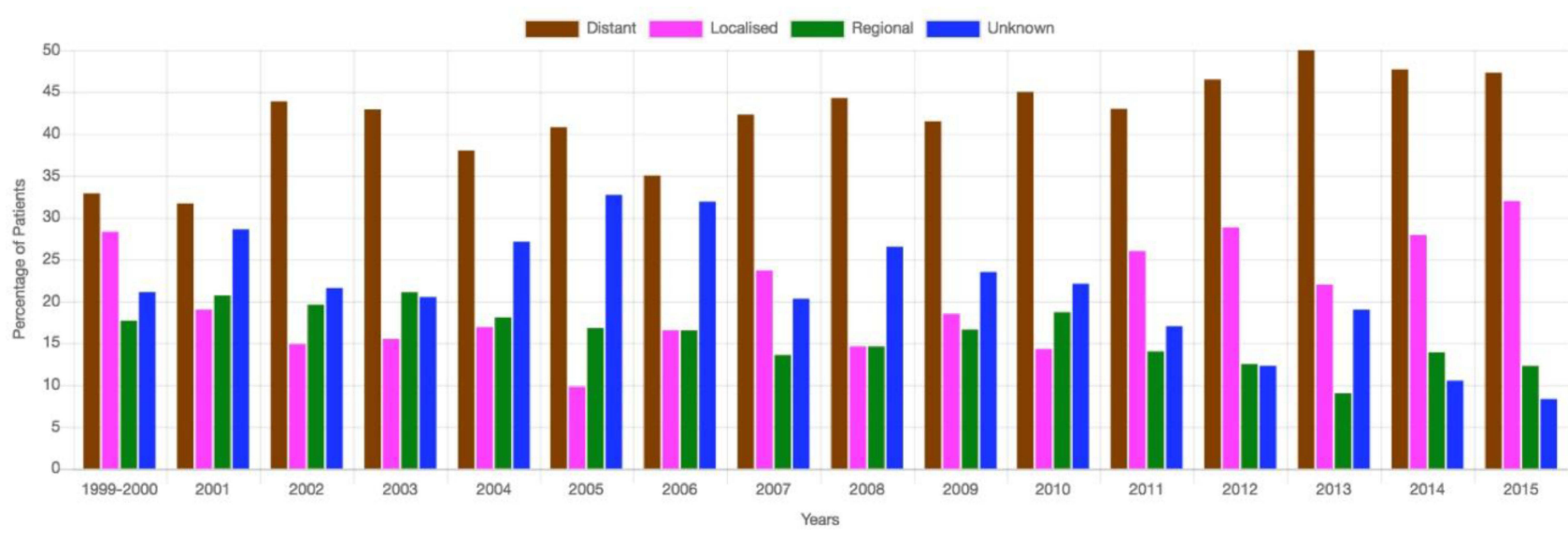

Figure 6 Stage distribution of NHL in Saudi patients. The percentage of stage distribution of NHL that were reported in the 17 years was compared.

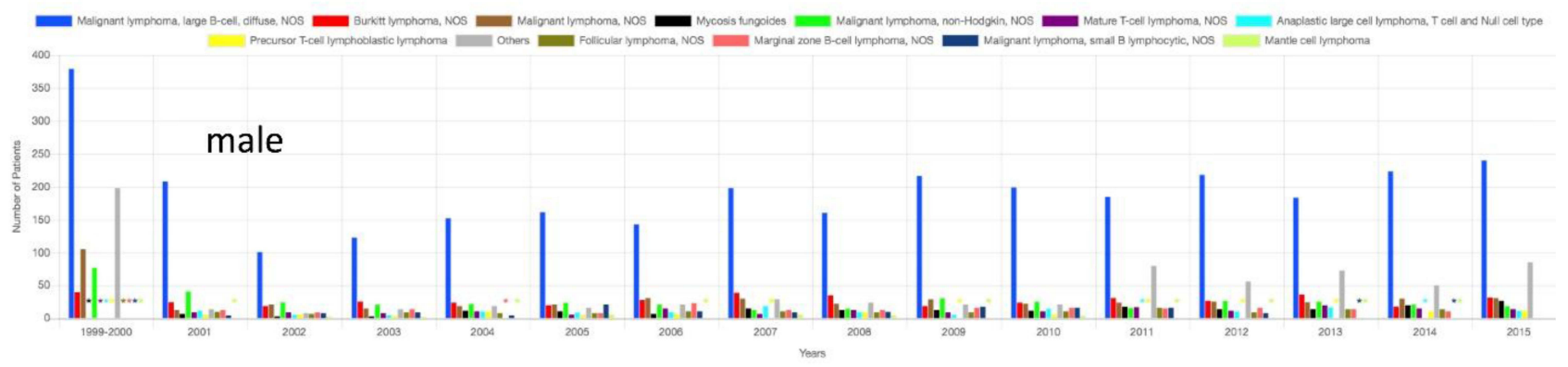

Figure 7 Morphology distribution of NHL in Saudi patients. CSA application was used to compare the number of patients assigned to an NHL morphology that were reported in all the 17 years. Asterisk symbol was used to indicate non-available data from SCIRs.

\section{Discussion}

IT holds a promise as enablers to easily and timeeffectively study and manage big data in health care. ${ }^{3}$ Various web-based applications have been constructed to allow a fast, intuitive, and interactive method for working with cancer data. For example, the cancer statistics data visualizations tool (CSDVT; https://gis.cdc.gov/Cancer/ USCS/DataViz.html) accommodates American-related cancer data resourced from the American Center for Disease Control and Prevention (CDC) and the National Cancer Institute (NCI). ${ }^{19}$ Another example is CancerData (https://www.cancerdata.nhs.uk/) that presents cancer data of patients living in England, which are gathered from the National Cancer Registration and Analysis Service (NCRAS). ${ }^{20}$ Encouraged by these two examples and due to the urgent demand for a simple and quick method to study Saudi cancer data in the SCIRs, we developed CSA to be the first web-based tool in Saudi Arabia to study SCIRs data. Although the English language is common among people working in healthcare and in the academic sectors, we have made CSA available in two languages, Arabic and English, to make our application approachable for non-English speakers. As a user-friendly and fast application, we believe that CSA will be useful tool to study cancer data among Saudis and will make the data published in SCIRs more reachable and usable.

There is a resemblance between CSA and the other two applications (CSDVT and CancerData) with respect to the tasks they conduct. Nevertheless, there are some specific features of CSDVT and Cancer Data that are not available in CSA, such as the analysis of survival rate of cancer patients. Information about survival rate is a key to evaluating the quality of healthcare services provided to patients diagnosed with tumors. It is also an indication of whether a type of cancer is life-threatening. Due to the limited survival data of Saudi cancer patients in SCIRs, the analysis of survival rate was not included in CSA. In fact, only a single SCIR that was published in 2007 reported survival data from 1994 to 2004 for only 5141 of 65,873 Saudi patients who were diagnosed in the same time 

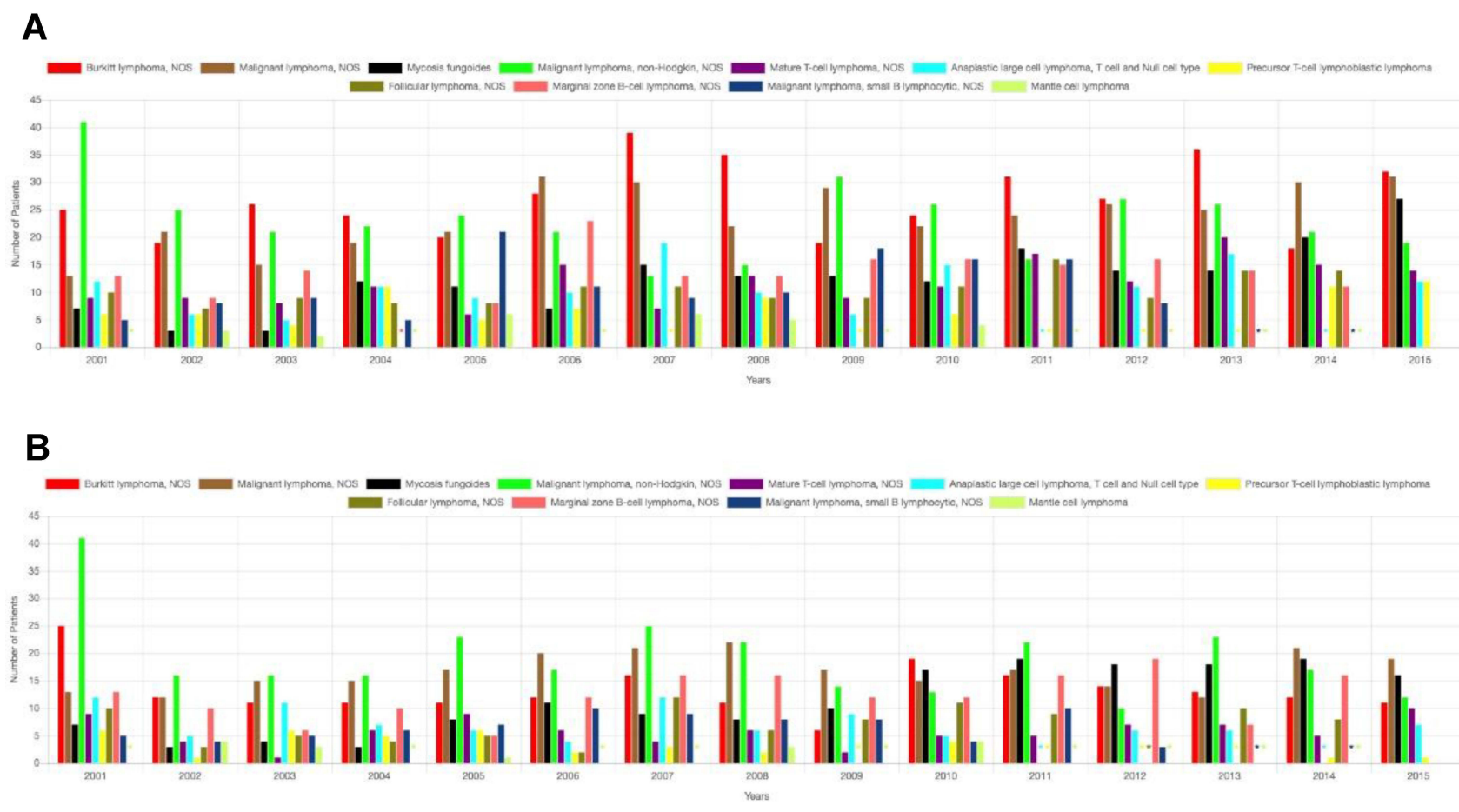

Figure $\mathbf{8}$ The less frequent morphologies of NHL in Saudi patients. The non-common morphologies of NHL amongst Saudi patients were compared in males (A) and females (B). Asterisk symbol was used to indicate non-available data from SCIRs.

period. We believe that publishing the survival data in a regular basis for all diagnosed patients will add a significant value to the knowledge provided by SCIRs. In contrast to CSDVT and Cancer Data, CSA allows the study of cancer stages and compares the ASR of cancer incidents in Saudis with that of patients from other countries. Cancer stage is a very important determinant for the time and type of treatment a patient should receive. Generally, patients at early stages of cancer do not receive as early and intensified therapy as those at the later stages of the disease. Therefore, the analysis titled "stage distribution of cancer" in CSA helps to gain knowledge about cancer stage of particular tumors in Saudi Arabia. Furthermore, it answers the question as to whether healthcare systems are able to make an early detection of a tumor, which in turn impacts positively on the success of therapy.

NHL is a hematological cancer that comprises different subtypes with variable pathology and clinical outcomes. ${ }^{21}$ NHL was selected in the present study as an example of SCIRs data to illustrate how CSA works because it is a common tumor; NHL is the second and fifth most prevalent neoplasm in Saudi males and females, respectively. ${ }^{7}$ NHL is considered a disease of the elderly with male to female prevalence. ${ }^{20}$ Our findings fit with this; NHL showed greater prevalence in older patients with larger incidence rates in males compared with females. Interestingly, our findings indicated a dramatic rise of NHL prevalence in the Riyadh and Eastern regions compared with the Jazan region. Although there is no definite explanation for the high ASR of NHL in the Riyadh and Eastern regions, risk factors may play a role in this observation. Therefore, screening for risk factors of NHL in the regions of Riyadh and Eastern may be worthwhile in explaining the predominance of the disease in these two regions. Developed countries recorded higher ASR of NHL compared with developing countries. ${ }^{22}$ Similarly, our analyses showed bigger ASR of NHL from USA compared with that of Saudi Arabia and the other Arabic countries. We found that the largest proportion of NHL patients have been diagnosed at the late stage (stage: distance). Consistently, the data of SEER from 2005 to 2015 reported that $50 \%$ of NHL patients have been diagnosed at the late stage. ${ }^{23}$ DLBCL is the most common type of NHL accounting for $58 \%$ of NHL series. ${ }^{24,25}$ Our results reached a similar conclusion, where DLBCL dominated the other types (morphologies) of NHL in Saudis. Taken together, our NHL findings provide an almost comprehensive view of the epidemiology of the disease in Saudis for 17 years. 
Currently, CSA accommodates the data of three different kinds of cancer: breast cancer, leukemia, and NHL. Our future plan is to cover the remaining malignant diseases by feeding our CSA with the data of other neoplasms from SCIRs. In addition, work will continue with the authors of SCIRs to obtain survival data of cancer patients in order to develop a new function of CSA that conducts survival analysis similar to that performed by CSDVT and Cancer Data. Effort will be also made to employ artificial intelligence to CSA in order to predict the cancer epidemiology in Saudis in the future.

\section{Conclusion}

Overall, we develop CSA to meet the great demand of a user-friendly tool to study the big data of cancer in Saudis reported in SCIRs. As a result, CSA enables reaching, extraction, comparison, and visualization of cancer data in SCIRs in an interactive, easy, and fast manner. Using CSA, we studied the data of NHL in Saudis reported in 16 SCIRs. Therefore, our findings painted an almost complete picture of the epidemiology of NHL in Saudis for 17 years (1999-2015). As an easy and timeeffective tool, we believe that CSA will be useful for people working in healthcare and academic sectors.

\section{Funding}

This research received no external funding.

\section{Disclosure}

Dr Suliman A Alsagaby and Dr Mafawez $\mathrm{T}$ Alharbi report patent copyrights (20-1-0001705) issued by Saudi Authority for Intellectual Property to the software and web-based tools of the website named "Cancer in Saudi Arabia" (https://cancerinsaudi.com/). The cancer data shown in the website of "Cancer in Saudi Arabia" have been authored and published by the Saudi Cancer Registry - Saudi Health Council - Ministry of Heath in the Kingdom of Saudi Arabia. Permission to use the cancer data reported in the Saudi Cancer Incidence Reports (SCIRs) was granted to the authors by the Saudi Cancer Registry - Saudi Health Council. The authors report no conflicts of interest in this work.

\section{References}

1. Raghupathi W, Raghupathi V. Big data analytics in healthcare: promise and potential. Health Info Sci Syst. 2004;2(1):1-10.

2. Stylianou A, Talias M. Big data in healthcare: a discussion on the big challenges. Health Technol (Berl). 2017;7(1):97-107. doi:10.1007/ s12553-016-0152-4
3. Murdoch T, Detsky A. The inevitable application of big data to health care. JAMA. 2013;309(13):1351-1352. doi:10.1001/jama.2013.393

4. Bennett T, Callahan T, Feinstein J, et al. Data science for child health. J Pediatr. 2019;208:12-22. doi:10.1016/j.jpeds.2018.12.041

5. Sung H, Ferlay J, Siegel RL, et al. Global cancer statistics 2020: GLOBOCAN estimates of incidence and mortality worldwide for 36 cancers in 185 countries. CA Cancer J Clin. 2021;71(3):209-249.

6. Siegel RL, Miller KD, Jemal A. Cancer statistics 2015. CA Cancer J Clin. 2015;65(1):5-29.

7. Cancer incidence report from Saudi Arabia 2015; 2018. Available from: https://nhic.gov.sa/en/eServices/Documents/2015.pdf. Accessed December 20, 2020.

8. Pressman RS. Software Engineering: A Practitioner's Approach. Palgrave macmillan; 2005.

9. Alshamrani A, Bahattab A. A comparison between three SDLC models waterfall model, spiral model, and incremental/iterative model. Int J Comp Sci Issues. 2015;12(1):106.

10. Rezende A, Silva L, Britto A, Amaral R. Software project scheduling problem in the context of search-based software engineering: a systematic review. J Syst Softw. 2019;155:43-56. doi:10.1016/j. jss.2019.05.024

11. Hölldobler K, Rumpe B, Wortmann A. Software language engineering in the large: towards composing and deriving languages. Comp Lang Syst Struct. 2018;54:386-405. doi:10.1016/j.cl.2018.08.002

12. Beighley L, Morrison M. Head First PHP \& MySQL: A BrainFriendly Guide. 1st ed. Sebastopol: O'Reilly Media; 2008.

13. Stauffer M. Laravel: Up \& Running: A Framework for Building Modern PHP Apps of Entry. 2nd ed. Sebastopol: O'Reilly Media; 2019.

14. Powell T. HTML \& CSS: The Complete Reference of Entry. 1st ed. New York: McGraw-Hill; 2010.

15. Rauschmayer A. Speaking JavaScript: An In-Depth Guide for Programmers of Entry. 1st ed. Sebastopol: O'Reilly Media, Inc.; 2014.

16. Nelson B. Getting to Know Vue. Js of Entry. 1st ed. New York: Springer; 2018.

17. Young J. SEER Summary Staging Manual 2000: Codes and Coding Instructions of Entry. Bethesda: National Cancer Institute, National Institutes of Health; 2001.

18. Jack A, Percy C, Shanmugarathan S, Whelan S. International Classification of Diseases for Oncology: ICD-O of Entry. Third ed. Geneva: World Health Organization; 2000.

19. U.S. Cancer Statistics Working Group. U.S. cancer statistics data visualizations tool. November 2018 submission data (1999-2016): U.S. Department of Health and Human Services, Centers for Disease Control and Prevention and National Cancer Institute; 2019. Available from: http://www.cdc.gov/cancer/dataviz. Accessed January 12, 2020.

20. CancerData. Available from: http://www.cancerdata.nhs.uk/. Accessed January 10, 2020.

21. Shankland K, Armitage J, Hancock B. Non-Hodgkin lymphoma. Lancet. 2012;380(9844):848. doi:10.1016/S0140-6736(12)60605-9

22. Müller A, Ihorst G, Mertelsmann R, Engelhardt M. Epidemiology of non-Hodgkin's lymphoma (NHL): trends, geographic distribution, and etiology. Ann Hematol. 2005;84:1. doi:10.1007/s00277-0040939-7

23. Cancer stat facts: non-Hodgkin lymphoma. Available from: https:// seer.cancer.gov/statfacts $/ \mathrm{html} / \mathrm{nhl}$.html. Accessed December 5, 2020.

24. Sant M, Allemani C, Tereanu C, et al. Incidence of hematologic malignancies in Europe by morphologic subtype: results of the HAEMACARE project. $J$ Am Soc Hematol. 2010;116 (19):3724-3734.

25. Temmim L, Baker H, Amanguno H, Madda J, Sinowatz F. Clinicopathological features of extranodal lymphomas: Kuwait experience. Oncology. 2004;67:382. doi:10.1159/000082922 


\section{Publish your work in this journal}

The Journal of Multidisciplinary Healthcare is an international, peerreviewed open-access journal that aims to represent and publish research in healthcare areas delivered by practitioners of different disciplines. This includes studies and reviews conducted by multidisciplinary teams as well as research which evaluates the results or conduct of such teams or healthcare processes in general. The journal covers a very wide range of areas and welcomes submissions from practitioners at all levels, from all over the world. The manuscript management system is completely online and includes a very quick and fair peer-review system. Visit http://www.dovepress.com/testimonials. php to read real quotes from published authors. 